\title{
Impact of the dark rim artifact on quantification of myocardial perfusion and perfusion reserve from ssfp first pass images Raphael D Hazel*, Nathaniel Reichek and Yi Wang
}

Address: St Francis Hospital, Roslyn, NY, USA

* Corresponding author

from 13th Annual SCMR Scientific Sessions

Phoenix, AZ, USA. 21-24 January 2010

Published: 21 January 2010

Journal of Cardiovascular Magnetic Resonance 20 I0, I2(Suppl I):P253 doi:I0.I I86/I532-429X-I2-SI-P253

This abstract is available from: http://jcmr-online.com/content/I2/SI/P253

(c) 2010 Hazel et al; licensee BioMed Central Ltd.

\section{Introduction}

The measurement of myocardial perfusion by magnetic resonance imaging can be confounded by the Dark Rim Artifact (DRA). It appears as signal loss in a rim of pixels in the subendocardium at the boundary between the myocardium and LV blood. It has been shown that the DRA has similar severity in stress and rest perfusion images and occurs most frequently in images acquired with the Steady State Free Precession (SSFP) pulse sequence $(1,2)$.

\section{Purpose}

The purpose of this study is to determine the impact of the DRA on the quantification of absolute stress and rest myocardial perfusion and perfusion reserve using the SSFP pulse sequence.

\section{Methods}

We studied 5 volunteers without know coronary artery disease ( 2 males) recruited with IRB consent. In each case, perfusion imaging was performed with Gadodiamide $0.05 \mathrm{mM} / \mathrm{kg}$, at a rate of $6 \mathrm{ml} / \mathrm{s}$ and with a saturation recovery SSFP pulse sequence under adenosine $(140 \mu \mathrm{g} /$ $\mathrm{kg}$ ) induced stress and rest conditions, with a 20-minute washout period. The temporal resolution is $160 \mathrm{~ms}$ and spatial resolution is $2 \times 3 \times 8 \mathrm{~mm} 3$. Three rotational long axis images (HLA, VLA, and LVOT views) were acquired. A Fermi model was used to quantify regional dynamic contrast signal derived from two sets of myocardial contours, excluding the DRA in the first instance then including the DRA in the second data set, using Medis V2006 and a custom Matlab (The MathWorks, Natick, MA) perfusion analysis program. Dynamic signal intensity changes were measured for $30 \%, 50 \%$ and $(100 \%)$ of the myocardial as defined in Medis. The three long axis images was divided into six equal segments for analysis giving a total of 18 sectors for each volunteer.

\section{Results}

Figure 1 shows the changes in the signal intensity versus time curves for one sector containing 30\% of the myocardium, for stress and rest with and without the DRA. The most significant change occurs the perfusion reserve which decreases by $21.7 \%$ when the DRA is included, relative to the measurement when it is excluded. Absolute stress perfusion decreases by $-4.2 \%$ while rest perfusion increases by $17.6 \%$. Figure 2 gives a summary of the changes in absolute perfusion and perfusion reserve for $30 \%, 50 \%$ and $100 \%$ of the myocardium.

\section{Conclusion}

The DRA has a significant impact on the MPR causing it to decrease. The absolute rest perfusion increases while the stress perfusion decreases only slightly relative to when the DRA is excluded form the measurement. 

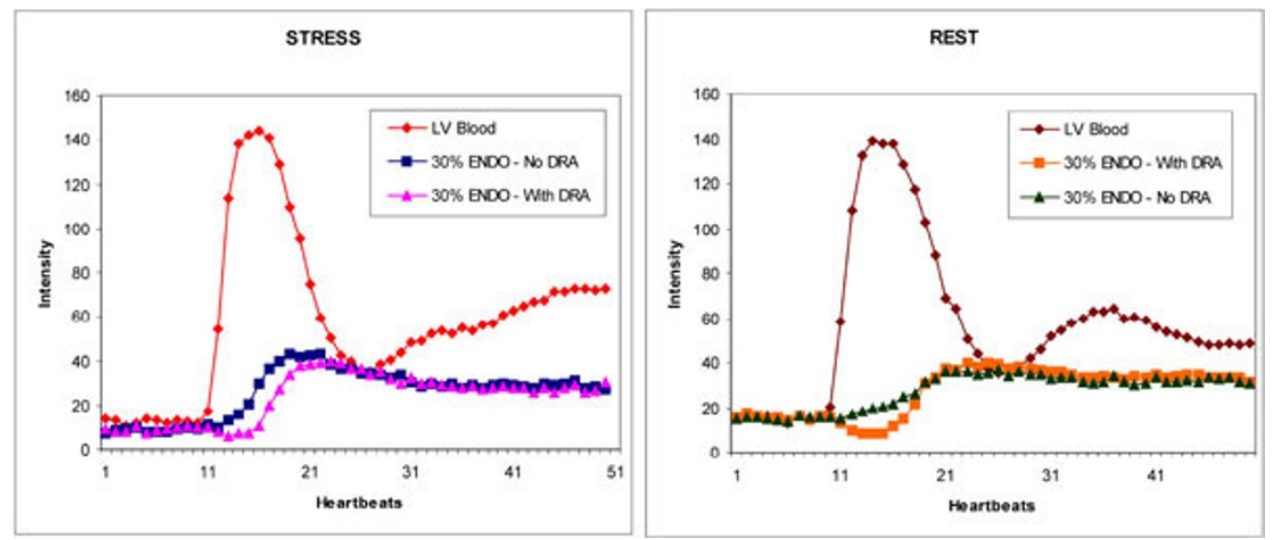

Figure I

Graph showing the change in signal intensity versus time for stress (left) and rest (right) images for blood and endocardium (30\%) including the excluding the dark rim artifact for a single case.

\begin{tabular}{|c|c|c|c|c|c|c|c|}
\hline \multicolumn{8}{|c|}{ Percentage Change in Perfusion when DRA is included } \\
\hline \multicolumn{3}{|c|}{ Extent of Myocardium } & Mean & SD & Max & Min & $\mathrm{p}$ value \\
\hline \multirow{3}{*}{\multicolumn{2}{|c|}{ MPR 30\% }} & NoDRA & 5.4 & 7.8 & 28.3 & 1.0 & \\
\hline & & DRA incl. & 3.9 & 5.1 & 17.7 & 0.6 & \\
\hline & & $\%$ Change & -21.7 & 14.2 & -2.4 & -43.7 & 0.06 \\
\hline \multirow{3}{*}{\multicolumn{2}{|c|}{ MPR $50 \%$}} & NODRA & 5.9 & 8.8 & 31.6 & 1.0 & \\
\hline & & DRA incl. & 4.2 & 5.7 & 19.1 & 0.7 & \\
\hline & & $\%$ Change & -22.7 & 18.1 & 8.6 & -55.1 & 0.06 \\
\hline \multirow{3}{*}{\multicolumn{2}{|c|}{ MPR $100 \%$}} & NoDRA & 7.2 & 11.2 & 40.2 & 1.0 & \\
\hline & & DRA incl. & 5.4 & 8.0 & 26.0 & 0.8 & \\
\hline & & $\%$ Change & -18.8 & 12.4 & -1.1 & -37.8 & 0.07 \\
\hline \multirow{3}{*}{ Stress } & $30 \%$ & $\%$ Change & -4.2 & 13.0 & 25.2 & -4.2 & 0.13 \\
\hline & $50 \%$ & $\%$ Change & -6.6 & 10.3 & 7.8 & -6.6 & 0.02 \\
\hline & $100 \%$ & $\%$ Change & -2.7 & 8.0 & 17.3 & -2.7 & 0.50 \\
\hline \multirow{3}{*}{ Rest } & $30 \%$ & $\%$ Change & 17.6 & 27.6 & 72.8 & 17.6 & 0.25 \\
\hline & $50 \%$ & $\%$ Change & 21.2 & 29.2 & 70.1 & 21.2 & 0.12 \\
\hline & $100 \%$ & $\%$ Change & 10.9 & 14.0 & 32.8 & 10.9 & 0.05 \\
\hline
\end{tabular}

Figure 2

Summary of the changes in myocardial perfusion reserve; and absolute stress and rest perfusion when the DRA is included in perfusion quantification relative to its exclusion DRA measurments were made for $\mathrm{m}=16$ sectors out of the total 90 sectors. 